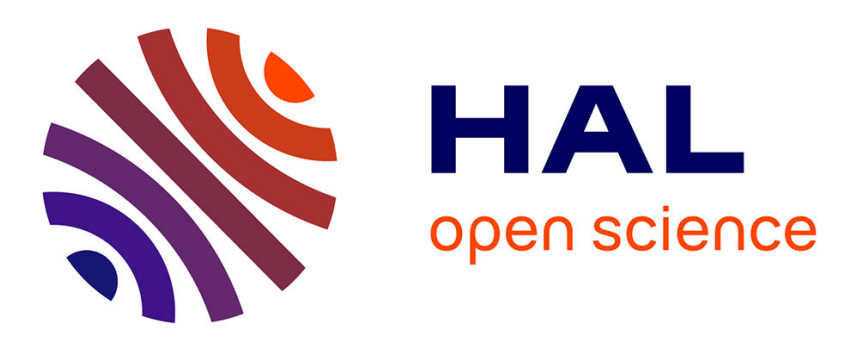

\title{
On the origin of a strong central peak in the local density of $d$ states at the surface of transition metals
}

J. Friedel

\section{To cite this version:}

J. Friedel. On the origin of a strong central peak in the local density of d states at the surface of transition metals. Journal de Physique, 1976, 37 (7-8), pp.883-894. 10.1051/jphys:019760037078088300 . jpa-00208483

\section{HAL Id: jpa-00208483 https://hal.science/jpa-00208483}

Submitted on 1 Jan 1976

HAL is a multi-disciplinary open access archive for the deposit and dissemination of scientific research documents, whether they are published or not. The documents may come from teaching and research institutions in France or abroad, or from public or private research centers.
L'archive ouverte pluridisciplinaire HAL, est destinée au dépôt et à la diffusion de documents scientifiques de niveau recherche, publiés ou non, émanant des établissements d'enseignement et de recherche français ou étrangers, des laboratoires publics ou privés. 


\title{
ON THE ORIGIN OF A STRONG CENTRAL PEAK IN THE LOCAL DENSITY OF d STATES AT THE SURFACE OF TRANSITION METALS
}

\author{
J. FRIEDEL \\ Physique des Solides $\left({ }^{*}\right)$, Université Paris-Sud, 91405 Orsay, France
}

(Reçu le 6 janvier 1976, accepté le 18 mars 1976)

\begin{abstract}
Résumé. - Le pic central observé sur les surfaces $(100)$ du Mo et du W ne peut pas être considéré comme dû à une anomalie de van Hove de la bande $\mathrm{d}$ du plan atomique de surface, élargie par interaction avec le volume du métal. On propose de le considérer comme dû à des états d virtuels liés aux atomes de surface et élargis par interaction avec le volume du métal. On souligne, dans ce contexte, une différence fondamentale entre surfaces compactes et moins compactes. On considère aussi d'autres cas où des pics de surface ont été calculés dans l'approximation de Huckel.

Abstract. - The central peak observed on the (100) surfaces of Mo and W cannot be thought of as due to a van Hove anomaly of the $d$ band of the surface atomic plane, broadened by interaction with the volume of the metal. It is argued that it might be considered as due to virtual $\mathrm{d}$ states on the surface atoms, broadened by interaction with the volume of the metal. A basic difference between closepacked and not so closepacked surfaces in stressed in this connection. Other cases where surface peaks have been computed in the LCAO approximation are also considered.
\end{abstract}

1. Introduction. - Various tight binding (LCAO) studies of the density of states of the $d$ band, for different types of surfaces [1 to 8$]\left({ }^{1}\right)$, have lead to the prediction that there should be a central peak of loeal density of states, near the middle of the $\mathrm{d}$ band on the surface atoms of transitional metals. Photoemission studies of (BCC) Mo and W, with UV light in the range of 10 to $15 \mathrm{eV}$, show indeed a strong emission for (100) faces which can be attributed to a large and narrow peak of local density of states on the surface atoms just below the Fermi level; photoemission of (110) faces seem to show no such well defined and narrow peak [9-13] $\left({ }^{2}\right)$.

This experimental peak was first attributed to the existence of a surface state within a gap of the (total) band structure, opened by the spin orbit coupling $[7,8]$. However more recent computations using the simple tight binding approach for the $d$ band alone obtain a central peak on some surfaces, and especially the (100) plane of the BCC structure, whether spin orbit coupling is included or not; indeed it is less marked for larger spin orbit couplings; in these computations, the central peak falls within the $\mathrm{d}$ band and does not

(*) Laboratoire associé au C.N.R.S.

( ${ }^{1}$ ) Also Desjonquères, M. C. and Cyrot-Lackmann, F., private communication.

$\left(^{2}\right)$ Also Petroff, Y. and Lecante, J., private communication. seem necessarily bound to a band gap [3 to 7] $\left({ }^{1}\right)$. Also the experimental peak is very similar in Mo and W, with widely different spin orbit coupling [13]. Our poor knowledge of the transition probabilities for photoemission, owing to complications due to surface and many body effects, does not at present allow us to use the variation of the photoemission peak with photon energy to argue in favour or against the existence of a surface state [13]. The occurrence of a central peak seems however too frequent and not sensitive enough to the strength of spin orbit coupling for this type of explanation to hold generally. We shall not discuss it here. Indeed the types of explanations explored here are not dependent on whether the peak is related to the existence of a surface state or to a mere increase of the density of (volume) states on the surface atoms.

Two such types of explanations have been put forward for a central peak of the surface density of states :

- A van Hove singularity of some d sub-band of the surface atomic plane, broadened by its interactions with the $\mathrm{d}$ band of the volume atoms [6].

- Resonant (virtual) bound d states on the atoms of the surface plane weakly coupled with the $d$ band of the volume atoms [3-5] $\left.{ }^{1}\right)$.

It is the purpose of this paper to study the conditions 
of validity of either model. Using arguments deduced from simple models, we want to show that the central peak observed on the (100) surface of BCC metals cannot possibly be interpreted as a broadened van Hove singularity, but that it might be better described in terms of virtual d states on surface atoms. For this second model to apply, an essential condition is that the surface atomic plane is not too closepacked. Other cases are also discussed.

2. Broadened two dimensional van Hove singularity. - This type of explanation requires two conditions :

- The surface plane should have a central van Hove singularity.

- The broadening due to interactions with the rest of the lattice should be small.

We wish to show for the example of a simple s band that these conditions are not easy to obtain. We shall then analyse the more complex $\mathrm{d}$ bands of transitional metals.

2.1 Narrow s BAND. - 2.1.1 Plane lattice. Consider a narrow $\mathrm{s}$ band for a plane rectangular lattice (Fig. 1).
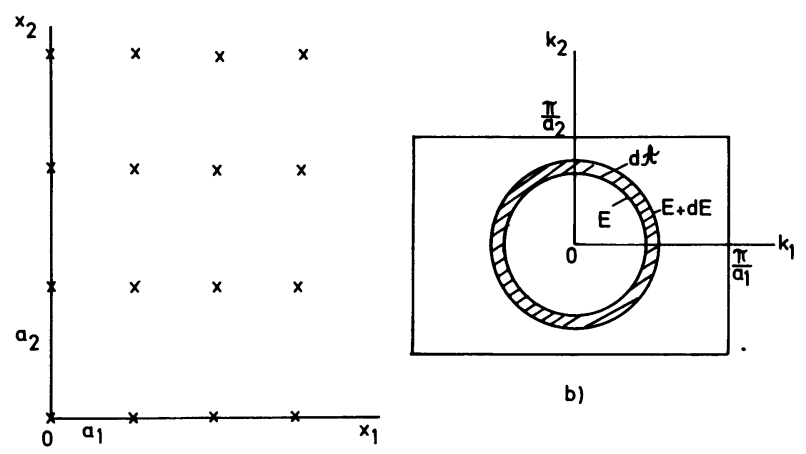

a)

FIG. 1. - Plane rectangular lattice : a) In real space; $b$ ) In reciprocal space.

In the usual simplest Huckel approximation, the energy $E$ of a Bloch state with wave vector $\mathbf{k}\left(k_{1}, k_{2}\right)$ can be written

$$
E=E_{1}\left(k_{1}\right)+E_{2}\left(k_{2}\right)
$$

where, for $i=1,2$

$$
E_{i}=2 \beta_{i} \cos k_{i} a_{i}
$$

$\beta_{i}$ are the two characteristic (and negative) transfer integrals. The energy of the atomic s state is taken as the zero of energy.

The density of states $n^{\prime \prime}(E)$, per unit energy $E$, of the two-dimensional s band is such that

$$
n^{\prime \prime}(E) \mathrm{d} E=\int_{\mathrm{d} \mathcal{A}_{E}} n_{1}^{\prime}\left(k_{1}\right) \mathrm{d} k_{1} n_{2}^{\prime}\left(k_{2}\right) \mathrm{d} k_{2}
$$

where integration is over the states $\mathbf{k}$ of energy between $E$ and $E+\mathrm{d} E$. By definition,

$$
n_{i}^{\prime}\left(E_{i}\right) \mathrm{d} E_{i}=2 n_{i}^{\prime}\left(k_{i}\right) \mathrm{d}\left|k_{i}\right| .
$$

The factor 2 comes from the two states $\pm k_{i}$ with the same energy $E_{i}$. With eq. (1), this gives

$$
n^{\prime \prime}(E)=\int n_{1}^{\prime}\left(E^{\prime}\right) n_{2}^{\prime}\left(E-E^{\prime}\right) \mathrm{d} E^{\prime}
$$

Using the fact that, for a row of length $L$,

$$
n_{i}^{\prime}(E)=\frac{L}{2 \pi\left|\beta_{i}\right| a_{i}} \frac{1}{\sqrt{1-\left(E_{i} / 2 \beta_{i}\right)^{2}}},
$$

it is easy to deduce the general form of $n^{\prime \prime}(E)$.

We have to integrate the product of two functions such as (6), shifted by $E$ with respect to each other. Figures $2 a$ and $b$ show the limits of integration depend on whether $|E| \gtrless 2\left|\beta_{1}-\beta_{2}\right|$.

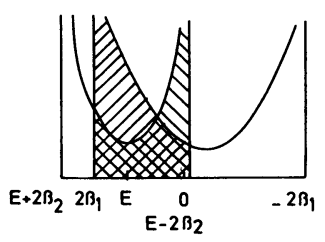

a)

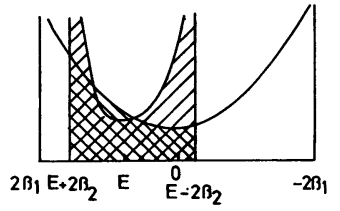

b)
Fig. 2. - Limits of integration of eq. (5) :a) $2 \beta_{1} \leqslant E^{\prime} \leqslant E-2 \beta_{2}$ if $\left.2\left(\beta_{1}+\beta_{2}\right)<E<2\left(\beta_{1}-\beta_{2}\right)<0 ; b\right) E+2 \beta_{2}<E^{\prime}<E-2 \beta_{2}$ if $2\left(\beta_{1}-\beta_{2}\right)<E<0$.

The band edges occur for $E= \pm 2\left|\beta_{1}+\beta_{2}\right|$. They have finite values

$$
\begin{array}{r}
n^{\prime \prime}\left( \pm 2\left|\beta_{1}+\beta_{2}\right|\right)=\lim _{\varepsilon \rightarrow 0} n^{\prime \prime}\left( \pm 2\left|\beta_{1}+\beta_{2}-\varepsilon\right|\right)= \\
=A \pi\left|\beta_{1} \beta_{2}\right|^{1 / 2},
\end{array}
$$

where

$$
A=L^{2} / 4 \pi^{2}\left|\beta_{1} \beta_{2}\right| a_{1} a_{2} .
$$

- If $\left|\beta_{1}\right| \neq\left|\beta_{2}\right|$, there are two logarithmic van Hove singularities at $E= \pm 2\left|\beta_{1}-\beta_{2}\right|$ (Fig. 3a) :

$n^{\prime \prime}(E) \simeq A \sqrt{\left|\beta_{1} \beta_{2}\right|} \ln |E \mp 2| \beta_{1}-\beta_{2}||$.

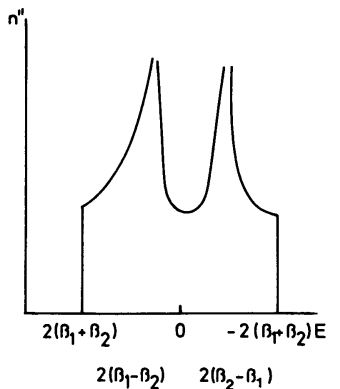

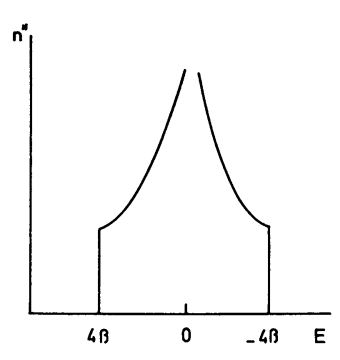

FIG. 3. - Density of states of the $s$ band for the atomic plane of figure $1: a)\left|\beta_{1}\right|>\left|\beta_{2}\right|\left(a_{1}<a_{2}\right.$, figure 1$\left.) ; b\right)\left|\beta_{1}\right|=\left|\beta_{2}\right|=|\beta|$ $\left(a_{2}=a_{1}\right.$, figure 1$)$. 
The density of states shows no anomaly at the center $E=0$ of the band.

- If $\left|\beta_{1}^{*}\right|=\left|\beta_{2}\right|=|\beta|$, the two van Hove anomalies merge into a single logarithmic peak at the center of the band (Fig. $3 b$ ) :

$$
\begin{array}{r}
n^{\prime \prime}(E)=A \int_{E+2 \beta}^{-2 \beta} \times \\
\times \frac{\mathrm{d} E^{\prime}}{\sqrt{\left[1-\left(\frac{E^{\prime}}{2 \beta}\right)^{2}\right]\left[1-\left(\frac{E^{\prime}-E}{2 \beta}\right)^{2}\right]}} \simeq \\
\simeq 4 A \beta \ln |E| .
\end{array}
$$

2.1.2 Three-dimensional density of states. - Let us now couple a collection of atomic planes such as in figure $1 a$, so as to form a three-dimensional quadratic structure where each atom of one plane has one effective neighbour in a neighbouring plane, with a transfer integral $\beta_{3}$ (Fig. 4).

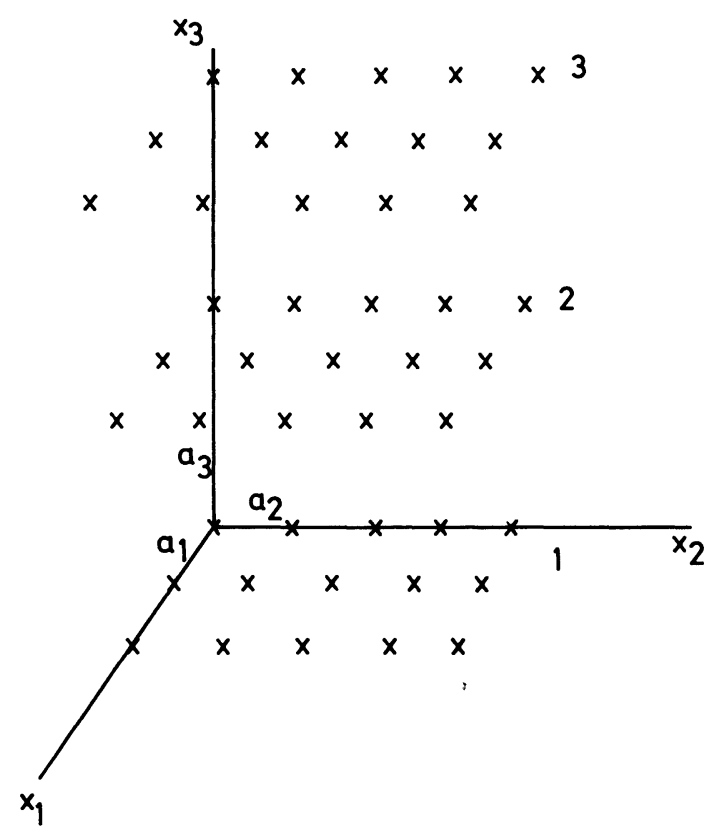

FIG. 4. - Simple quadratic structure.

A reasoning similar to that made above gives, for the (volume) density of states of the three-dimensional structure

$$
\begin{aligned}
n^{\prime \prime \prime}(E) & =\int n^{\prime \prime}\left(E^{\prime}\right) n_{3}^{\prime}\left(E-E^{\prime}\right) \mathrm{d} E^{\prime} \\
& =\int n_{1}^{\prime}\left(E^{\prime \prime}\right) n_{2}^{\prime}\left(E^{\prime}-E^{\prime \prime}\right) n_{3}^{\prime}\left(E-E^{\prime}\right) \mathrm{d} E^{\prime} \mathrm{d} E^{\prime \prime}
\end{aligned}
$$

where $n_{3}^{\prime}\left(E_{3}\right)$ is given by eq. (6).

The convolution of the two-dimensional density $n^{\prime \prime}\left(E^{\prime}\right)$ by the one-dimensional density $n_{3}^{\prime}\left(E-E^{\prime}\right)$ will produce a rounding of the band edges and alter the other van Hove anomalies.

- If $\left|\beta_{1}\right|=\left|\beta_{2}\right|=\left|\beta_{3}\right|=|\beta|$, one obtains the well known two van Hove anomalies of the simple cubic structure (Fig. $5 a$ ) : the coupling due to $\left|\beta_{3}\right| \neq 0$ splits the central peak into two less strongly marked and off centre singularities.

- If $\left|\beta_{1}\right|=\left|\beta_{2}\right| \neq\left|\beta_{3}\right|$ (and in the two other cases deduced by circular permutations of $1,2,3$ ), the coupling due to $\left|\beta_{3}\right|$ again splits the central peak and the edges of the band (Fig. $5 b$ ).
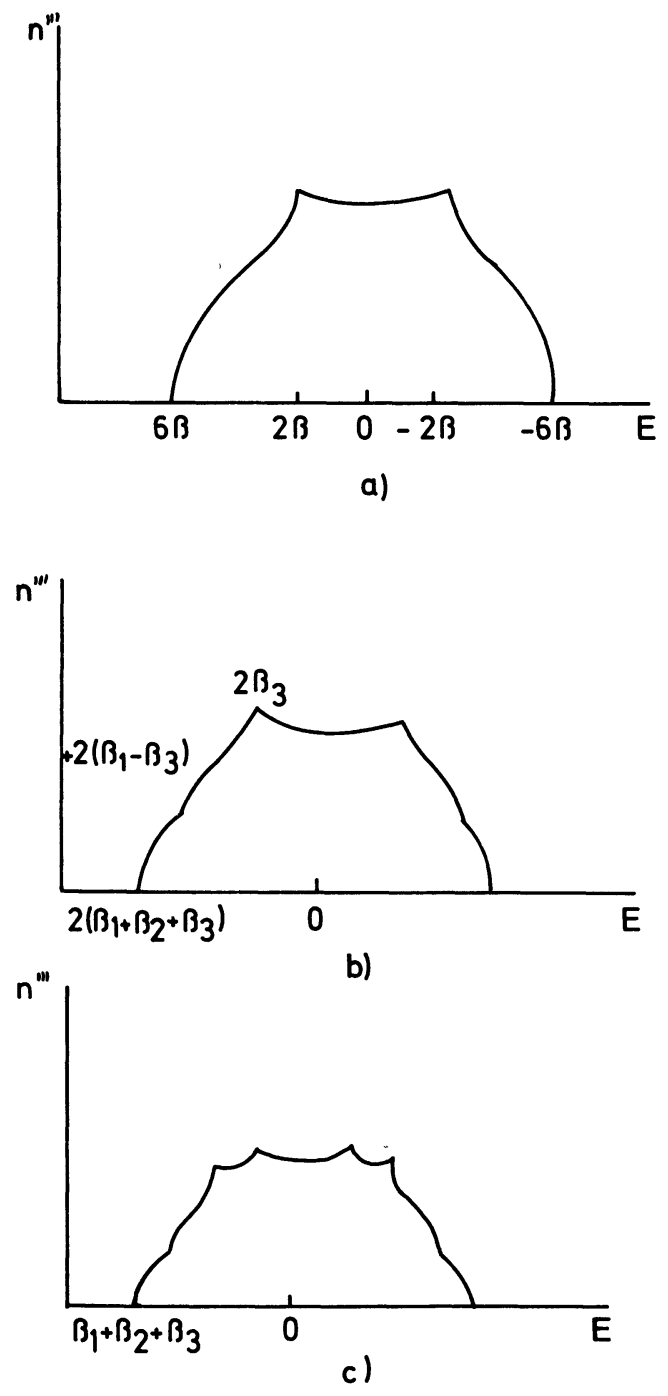

FIG. 5. - Volume density of states for the quadratic structure of figure 3 (schematic) :

a) $\left|\beta_{1}\right|=\left|\beta_{2}\right|=\left|\beta_{3}\right|=|\beta|$;

$$
\text { b) }\left|\beta_{1}\right|=\left|\beta_{2}\right| \neq\left|\beta_{3}\right|
$$

(or $\left|\beta_{2}\right|=\left|\beta_{3}\right| \neq\left|\beta_{1}\right|$, or $\left|\beta_{3}\right|=\left|\beta_{1}\right| \neq\left|\beta_{2}\right|$ );

c) $\left|\beta_{1}\right|,\left|\beta_{2}\right|,\left|\beta_{3}\right|$ all unequal : anomalies at $\pm \beta_{1} \pm \beta_{2} \pm \beta_{3}$.

- If $\left|\beta_{1}\right|,\left|\beta_{2}\right|$ and $\left|\beta_{3}\right|$ are all unequal, there are in general four van Hove singularities besides the band edges. For $\left|\beta_{3}\right|$ small, they can be thought of as a splitting by $\left|\beta_{3}\right|$ of each anomaly of figure $3 a$. 
2.1.3 Local density of states on the surface atoms. - We now consider a finite three-dimensional stacking such as in figure 4.

Writing the wave functions $|\psi(E)\rangle$ of the s band as linear combinations of two-dimensional Bloch functions $|n\rangle$, of wave vector $\mathbf{k}$, on each $x_{1} x_{2}$ atomic plane (denoted by its order $P$ )

$$
|\psi\rangle=\sum_{P} a_{P}|P\rangle,
$$

$|\psi\rangle$ can be thought of as the eigenfunction of the effective Hamiltonian

$$
\mathscr{H}_{3}=\beta_{3} \sum_{\substack{P \\ Q \text { near to } P}}|P\rangle\langle Q|,
$$

with eigenvalues

$$
E_{3}=E-\left[E_{1}\left(k_{1}\right)+E_{2}\left(k_{2}\right)\right] .
$$

The local density of states on atoms of plane $P$ is then

$$
\frac{\mathrm{d} \rho_{P}}{\mathrm{~d} E} \propto \int a_{P}^{2}\left(E^{\prime}\right) n_{3}\left(E^{\prime}\right) n^{\prime \prime}\left(E-E^{\prime}\right) \mathrm{d} E^{\prime} .
$$

For a collection of atomic planes $P=1$ to $N_{3}$ (Fig. 6), the solution of the linear chain problem (11), (12) is easily shown to be

with

$$
a_{P}\left(E_{3}\right)=\sqrt{\frac{2}{N_{3}+1}} \sin P k_{3} a_{3}
$$

$$
k_{3} a_{3}=q \pi /\left(N_{3}+1\right) \text {. }
$$

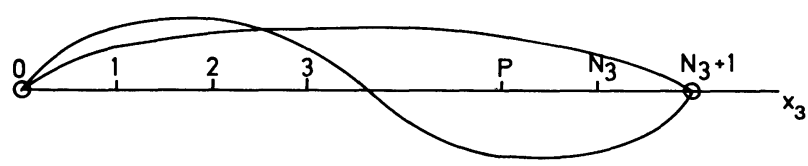

FIG. 6. - The linear chain problem

To compute $\mathrm{d} \rho_{P} / \mathrm{d} E$, one has therefore to convolute the two-dimensional density of states $n^{\prime \prime}\left(E-E^{\prime}\right)$ with a function proportional to $n_{3} \sin ^{2} P k_{3} a_{3}$. For the surface plane $P=1$, this is proportional to $\sin k_{3} a_{3}$ or $\sqrt{1-\left(E^{\prime} / 2 \beta_{3}\right)^{2}}$; for the second plane, this is proportional to $\sin ^{-1} k_{3} a_{3} \sin ^{2} 2 k_{3} a_{3}$, etc... For a plane deep in the volume, thus $P$ large, the function $\sin ^{-1} k_{3} a_{3} \sin ^{2} P k_{3} a_{3}$ tends towards $n_{3}\left(E^{\prime}\right)$. These functions are compared figure 7 (cf. [14]).

One can then discuss the effect of three-dimensional coupling on the local density of states $\mathrm{d} \rho_{P} / \mathrm{d} E$, in the same way as for the volume density of states.

The number and position of the van Hove singularities are the same. But on the surface plane $P=1$, the three-dimensional coupling due to $\beta_{3}$ produces a broadening rather than a splitting. Thus, if

$$
\left|\beta_{3}\right| \ll\left|\beta_{1}\right|=\left|\beta_{2}\right|,
$$

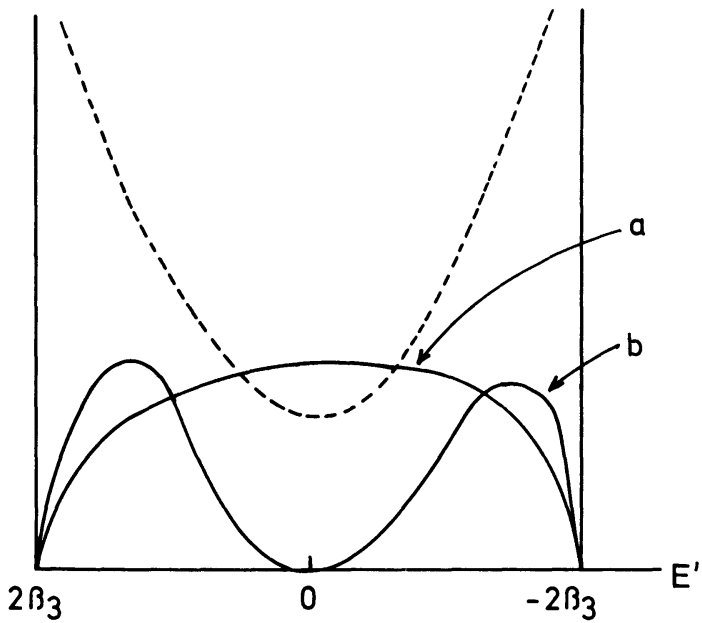

FIG. 7. - Convoluting functions :

a) $\sin k_{3} a_{3}$ and $\left.b\right) \sin k_{3} a_{3} \sin ^{2} 2 k_{3} a_{3}$, compared to function $n_{3}\left(E^{\prime}\right)$ (in broken line).

one expects the central peak of figure $3 b$ to be broadened but preserved in the density $\mathrm{d} \rho_{1} / \mathrm{d} E$ of the surface plane; it should be split but still marked on the second plane, and correspond to two van Hove singularities in the volume density of states. In all other cases, no sharp central peak is expected.

2.1.4 Conclusion. - This analysis of a simple $\mathrm{s}$ band shows two points :

- The two-dimensional density of states of an isolated atomic plane has a central peak only under very specific conditions. In the case of the rectangular array discussed here, the condition $\left|\beta_{1}\right|=\left|\beta_{2}\right|$ corresponds to a resonance effect in the convolution of the two component one-dimensional densities of states. No central peak is expected if $\left|\beta_{1}\right| \neq\left|\beta_{2}\right|$ or, as easily seen, in two-dimensional hexagonal close packed layers.

- If the isolated surface plane has a two-dimensional central peak, this is preserved, with some broadening, only if the interplanar coupling is smaller than the intraplanar ones. In that case, the central peak on the surface plane is replaced, in the parallel planes inside the crystal, by a double peak, split symmetrically with respect to the centre of the band, with a splitting of the same order as the width of the central peak at the surface.

In the extension that follows, account is taken of the fact that the results for densities of states do not depend on the sign of the transfer integrals $\beta_{i}$.

2.2 ExTENSION TO d BANDS. - For each pair of atoms, there are now different types of transfer integrals, usually denoted $\sigma, \pi$ and $\delta$ (Fig. 8). As a rule, for first neighbours, $-\sigma>\pi \gg-\delta>0$. Futhermore, in BCC lattices where second neighbours have to be taken into account,

$$
-\sigma_{2} \simeq+\pi_{1}>+\pi_{2} \gg-\delta_{2} \simeq 0 \quad[15]
$$




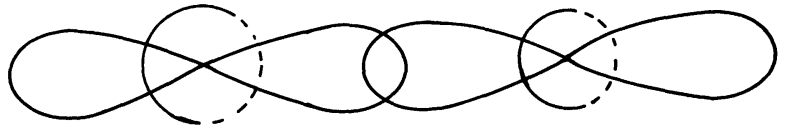

a)

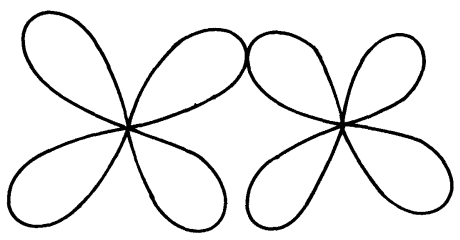

b)

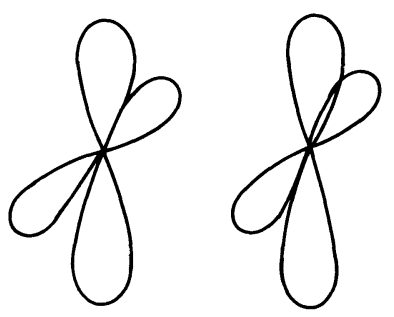

c)

FIG. 8. - Different types of dd transfer integrals : a) $\sigma ; b) \pi ; c$ ) $\delta$.

2.2.1 (100) surface of the BCC lattice. - The $\mathrm{d}$ functions can be classified, with obvious notations into $x_{1} x_{2}, x_{2} x_{3}, x_{3} x_{1}, x_{1}^{2}-x_{2}^{2}$, and $x_{33}$.

For a single atomic plane, with its square array of atoms (Fig. 9a), these atomic functions lead to 5 independent sub-bands. With second nearest neighbour interactions, three sub-bands of pure $x_{1} x_{2}, x_{2} x_{3}$, $x_{3} x_{1}$, character respectively, and two sub-bands of mixed $x_{1}^{2}-x_{2}^{2}$ and $x_{33}$ characters (cf. table I). It is easy to check that the $x_{1} x_{2}$ functions give a sub-band with a central peak, because their transfer integrals along $x_{1}$ and $x_{2}$ are equal. The $x_{2} x_{3}$ and $x_{3} x_{1}$ sub-bands have, on the contrary, no central peak, but two off centre peaks, at energies $\pm 2 \pi_{2}$ from the centre, because their transfer integrals along $x_{1}$ and $x_{2}$ are different. Finally a direct study of the mixed $x_{1}^{2}-x_{2}^{2}$ and $x_{33}$ sub-bands (Appendix A) shows that they do not have any central peak : this was expected, as the coupling between $x_{1}^{2}-x_{2}^{2}$ and $x_{33}$ orbitals on neighbouring atoms should split the central peak that the individual $x_{1}^{2}-x_{2}^{2}$ and $x_{33}$ sub-bands would have if uncoupled.

Now it is clear from table I and from the orders of

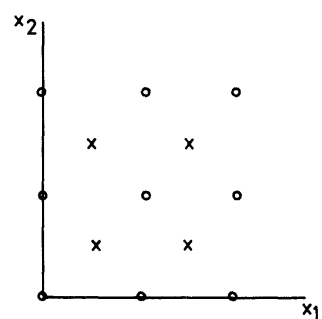

a)

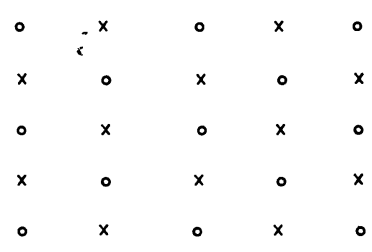

FIG. 9. - BCC structure : a) (100) surface; b) (110) surface. Circles : atoms of the first (surface) and third planes : crosses : atoms of the second plane.

TABLE I

Transfer integrals of $\mathrm{d}$ orbitals of surface atoms with atoms of the three first planes of a (100) surface in the BCC structure

\begin{tabular}{|c|c|c|c|c|c|c|}
\hline \multicolumn{2}{|c|}{$\mathrm{d}$ orbital of surface atoms } & $x_{33}$ & $x_{1}^{2}-x_{2}^{2}$ & $x_{1} x_{2}$ & $x_{2} x_{3}$ & $x_{3} x_{1}$ \\
\hline $\begin{array}{l}\text { Plane } 1 \\
\text { (4 neighbours) }\end{array}$ & $\begin{array}{c}x_{33} \\
x_{1}^{2}-x_{2}^{2} \\
x_{1} x_{2} \\
x_{2} x_{3} \\
x_{3} x_{1}\end{array}$ & $\begin{array}{c}{\left[\frac{\sigma+3 \delta}{4}\right]_{2}} \\
{\left[-\frac{\sqrt{3}}{4}(\sigma-\delta)\right]_{2}} \\
0 \\
0 \\
0\end{array}$ & $\begin{array}{c}{\left[-\frac{\sqrt{3}}{4}(\sigma-\delta)\right]_{2}} \\
{\left[\frac{3 \sigma+\delta}{4}\right]_{2}} \\
0 \\
0 \\
0\end{array}$ & $\begin{array}{c}0 \\
\\
0 \\
{[\pi]_{2}} \\
0 \\
0\end{array}$ & $\begin{array}{c}0 \\
0 \\
0 \\
0 \pi]_{2} \text { or }[\delta]_{2} \\
0\end{array}$ & $\begin{array}{c}0 \\
0 \\
0 \\
0 \\
0 \\
{[\pi]_{2} \text { or }[\delta]_{2}}\end{array}$ \\
\hline $\begin{array}{l}\text { Plane } 2 \\
\text { (4 neighbours) }\end{array}$ & $\begin{array}{c}x_{33} \\
x_{1}^{2}-x_{2}^{2} \\
x_{1} x_{2} \\
x_{2} x_{3} \\
x_{3} x_{1}\end{array}$ & $\begin{array}{c}{\left[\frac{2 \pi+\delta}{3}\right]_{1}} \\
{[0]} \\
{\left[-\frac{2 \sqrt{3}}{9}(\pi-\delta)\right]} \\
{\left[\frac{\sqrt{3}}{9}(\pi-\delta)\right]_{1}} \\
{\left[\frac{\sqrt{3}}{9}(\pi-\delta)\right]_{1}}\end{array}$ & $\begin{array}{c}{[0]} \\
{\left[\frac{2 \pi+\delta}{3}\right]_{1}} \\
{\left[-\frac{\pi+\delta}{3}\right]_{1}} \\
{\left[-\frac{\pi+\delta}{3}\right]_{1}}\end{array}$ & {$\left[\begin{array}{l}{\left[\begin{array}{l}-\frac{2 \sqrt{3}}{9}(\pi-\delta) \\
{[0]}\end{array}\right]} \\
{\left[\frac{3 \sigma+2 \pi-4 \delta}{9}\right]_{1}} \\
{\left[\frac{3 \sigma-\pi-2 \delta}{9}\right]_{1}} \\
{\left[\frac{3 \sigma-\pi-2 \delta}{9}\right]_{1}}\end{array}\right.$} & $\begin{array}{l}{\left[\frac{\sqrt{3}}{9}(\pi-\delta)\right]_{1}} \\
{\left[\frac{\pi+\delta}{3}\right]_{1}} \\
{\left[\frac{3 \sigma-\pi-2 \delta}{9}\right]_{1}} \\
{\left[\frac{3 \sigma+2 \pi+4 \delta}{9}\right]} \\
{\left[\frac{3 \sigma-\pi-2 \delta}{9}\right]}\end{array}$ & $\begin{array}{c}{\left[\frac{\sqrt{3}}{9}(\pi-\delta)\right]_{1}} \\
{\left[-\frac{\pi+\delta}{3}\right]_{1}} \\
{\left[\frac{3 \sigma-\pi-2 \delta}{9}\right]_{1}} \\
{\left[\frac{3 \sigma-\pi-2 \delta}{9}\right]_{1}} \\
{\left[\frac{3 \sigma+2 \pi+4 \delta}{9}\right]}\end{array}$ \\
\hline $\begin{array}{l}\text { Same orbital } \\
\text { (1 neighbour) }\end{array}$ & plane 3 & {$[\sigma]_{2}$} & {$[\delta]_{2}$} & {$[\delta]_{2}$} & {$[\pi]_{2}$} & {$[\pi]_{2}$} \\
\hline
\end{tabular}


magnitude of the various transfer integrals that the $x_{1} x_{2}$ orbitals have stronger and more numerous interactions with atomic orbitals centred on atoms of the second plane, where they have nearest neighbours, than within the surface plane, where they only have second nearest neighbours with $\pi_{2}$ overlap. The twodimensional $x_{1} x_{2}$ sub-band will therefore be much broadened, and the central peak effect should be essentially washed out.

In conclusion, we do not expect any van Hove anomaly of the $\mathrm{d}$ bands for the surface plane to lead to any central peak in the surface density of $d$ states on a (100) surface of the BCC structure.

On the contrary, full LCAO computations [4] give, in such a case, a central peak of the surface density of states, in agreement with photoemission experiments. In Mo, this peak has all the five $d$ characters, but is especially narrow and intense in the $x_{1}^{2}-x_{2}^{2}$ orbital. In $\mathrm{W}$, the larger spin orbit coupling has considerably washed out all but the $x_{1}^{2}-x_{2}^{2}$ peak. These results clearly show that the model of a two-dimensional van Hove anomaly cannot possibly apply in such a case.

2.2.2 Other cases. - Consider now a (110) surface of the BCC structure (Fig. 9b). It is immediately clear, without detailed analysis, that the directionality of the d-d transfer integrals makes it impossible for a rectangular lattice such as that of the surface plane to lead to the conditions $\left|\beta_{1}\right|=\left|\beta_{2}\right|$ necessary for a central peak. Indeed the surface densities obtained by full LCAO computations have no central peak. Possible double peaks due to two-dimensional van Hove anomalies fall within the two strong peaks of the volume density of states, and cannot be resolved. Therefore, for this more closepacked surface, the concept of a broadened two-dimensional van Hove anomaly might apply. But it does not lead to any very marked central peak.

A similar discussion can be made for the (100), (110) and (111) surfaces of the FCC structure, and for the (0001) surface of the HCP structure.

For one isolated atomic plane, one only expects a central peak for the $x_{1} x_{2}$ sub-band of the (100) surface in the FCC structure (Fig. 10 : note that we take $x_{1} x_{2}$ at $45^{\circ}$ to the axes of the cube). For the (100) and (110) surfaces of the FCC structure, the discussion is the same as for the same surfaces of the BCC structure. For the (111) surface of the FCC structure and the (0001) surface of the HCP structure, a fundamental asymmetry of the $\mathrm{d}$ sub-bands comes from the non alternate character of a hexagonal closepacked plane [19] : as the centre of the band has no special symmetry character, it can only be by chance that a van Hove anomaly could occur at the centre of the band.

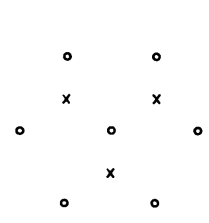

a)

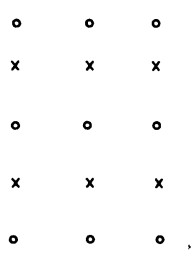

b)

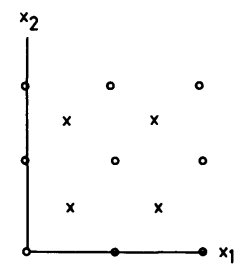

c)
FIG. 10. - Close packed structures : a) (111) surface of the FCC structure or (0001) surface of the HCP structure. b) (110) surface of the FCC structure. c) (100) surface of the FCC structure. Circles : atoms of the first (surface) plane; crosses : atoms of the second plane.

TABLE II

Transfer integrals of $\mathrm{d}$ orbitals of surface atoms with atoms of the two first planes of a (100) surface in the FCC structure

\begin{tabular}{|c|c|c|c|c|c|c|}
\hline \multicolumn{2}{|c|}{$\begin{array}{c}\mathrm{d} \text { orbitals } \\
\text { of surface atoms }\end{array}$} & $x_{33}$ & $x_{1}^{2}-x_{2}^{2}$ & $x_{1} x_{2}$ & $x_{2} x_{3}$ & $x_{3} x_{1}$ \\
\hline $\begin{array}{l}\text { Plane } 1 \\
\text { (4 neighbours) }\end{array}$ & $\begin{array}{c}x_{33} \\
x_{1}^{2}-x_{2}^{2} \\
x_{1} x_{2} \\
x_{2} x_{3} \\
x_{3} x_{1}\end{array}$ & $\begin{array}{c}{\left[\frac{\sigma+3 \delta}{4}\right]_{1}} \\
{\left[-\frac{\sqrt{3}}{4}(\sigma-\delta)\right]_{1}} \\
0 \\
0 \\
0\end{array}$ & {$\left[\begin{array}{c}-\frac{\sqrt{3}}{4}(\sigma-\delta) \\
{\left[\frac{3 \sigma+\delta}{4}\right]_{1}} \\
0 \\
0 \\
0\end{array}\right.$} & $\begin{array}{c}0 \\
0 \\
0 \\
{[\pi]_{1}} \\
0 \\
0\end{array}$ & $\begin{array}{c}0 \\
0 \\
0 \\
0 \\
{[\pi]_{1} \text { or }[\delta]_{1}} \\
0\end{array}$ & $\begin{array}{c}0 \\
0 \\
0 \\
0 \\
0 \\
{[\pi]_{1} \text { or }[\delta]_{1}}\end{array}$ \\
\hline $\begin{array}{l}\text { Plane } 2 \\
\text { (4 neighbours) }\end{array}$ & $\begin{array}{c}x_{33} \\
x_{1}^{2}-x_{2}^{2} \\
x_{1} x_{2} \\
x_{2} x_{3} \\
x_{3} x_{1}\end{array}$ & $\begin{array}{c}{\left[\frac{\sigma+12+3 \delta}{16}\right]_{1}} \\
{\left[\frac{\sqrt{3}}{16}(\sigma-4 \pi+3 \delta)\right]} \\
{\left[\frac{\sqrt{6}}{16}(\sigma-\delta)\right]_{1}} \\
{\left[\frac{\sqrt{6}}{16}(\sigma-\delta)\right]_{1}}\end{array}$ & $\begin{array}{c}{[0]} \\
{\left[\frac{\pi+\delta}{2}\right]_{1}} \\
{[0]} \\
{\left[-\frac{\sqrt{2}}{4}(\pi+\delta)\right]} \\
{\left[-\frac{\sqrt{2}}{4}(\pi+\delta)\right]_{1}}\end{array}$ & $\begin{array}{c}{\left[\frac{\sqrt{3}}{16}(\sigma-4 \pi+3 \delta)\right]} \\
{\left[\frac{3 \sigma+4 \pi+9 \delta}{16}\right]_{1}} \\
{\left[\frac{3 \sqrt{2}}{16}(\sigma-\delta)\right]_{1}} \\
{\left[\frac{3 \sqrt{2}}{16}(\sigma-\delta)\right]_{1}}\end{array}$ & 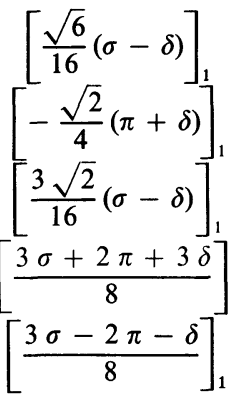 & $\begin{array}{l}{\left[\frac{\sqrt{6}}{16}(\sigma-\delta)\right]_{1}} \\
{\left[-\frac{\sqrt{2}}{4}(\pi+\delta)\right]_{1}} \\
{\left[\frac{3 \sqrt{2}}{16}(\sigma-\delta)\right]_{1}} \\
{\left[\frac{3 \sigma-2 \pi-\delta}{8}\right]_{1}} \\
-\end{array}$ \\
\hline
\end{tabular}


If now interactions between planes are taken into account, the central peak predicted for the $x_{1} x_{2}$ subband of the (100) surface in the FCC structure is again expected to broadened. The broadening should be somewhat less than in the corresponding case of the BCC structure, because the relative strengths of the intra and interplane interactions are different in the two cases (cf. tables I and II). But the broadening is still expected to be important, for the interplane interactions are more numerous and not much weaker than the intraplane ones.

Full d band LCAO computations [3-6] ( $\left.{ }^{1}\right)$ show indeed no central peak on closepacked surfaces ((111) of FCC, (0001) of HCP). They show however, contrary to these predictions, a well defined peak near to the centre of the band on the (100) and (110) surfaces of the FCC structure, with all but $x_{1}^{2}-x_{2}^{2}$ character. The computed peaks are shifted somewhat on the high energy side of the centre of the volume band, by an amount comparable to the shift computed for the whole surface density of states. This shift is clearly due to a repulsive correction to the atomic potentials on the surface plane, introduced for self-consistency in the metals of the end of the transitional series $(\mathrm{Ni}, \mathrm{Pt})$ where these computations have been made.

In conclusion, the model of a two-dimensional van Hove anomaly can explain the absence of a central peak on the most closepacked surfaces of the usual structures ((110) for BCC, (111) for FCC, (0001) for HCP). But it fails completely to explain the peaks observed or computed for not so closepacked surfaces ((100) for BCC, (110) and (100) for FCC).

3. Atomic virtual d levels. - It is then tempting to relate the central peaks to the atomic virtual bound d levels produced for single atoms adsorbed on surfaces. We shall now discuss this type of effect, using again simple models as a guide. We shall use the LCAO language throughout, and show that this point of view is an alternative simple interpretation of the full LCAO computations.

3.1 VIRTUAL BOUND d LEVEL OF A SINGLE ADSORBED AтOM. - When one transitional atom is adsorbed on a flat surface of the same metal, one expects and indeed computes that the local density of states on the adsorbed atom shows a strong central peak superimposed on a band with the same width as the volume $\mathrm{d}$ band (Fig. 11). This peak is easily understood as due to a small resonance broadening with the volume states of the local d states [16-18]. Again, because of the large density of $\mathrm{d}$ states in the region of interest, a simple LCAO description of volume and adsorbed $\mathrm{d}$ states is sufficient.

Indeed the width $w$ of the central peak is such that

$$
w \simeq \hbar / \tau_{\mathrm{a}},
$$

where $\tau_{\mathrm{a}}$ is the life time of an electron on an adsorbed atom. Thus $w$ is very roughly expected to be of the

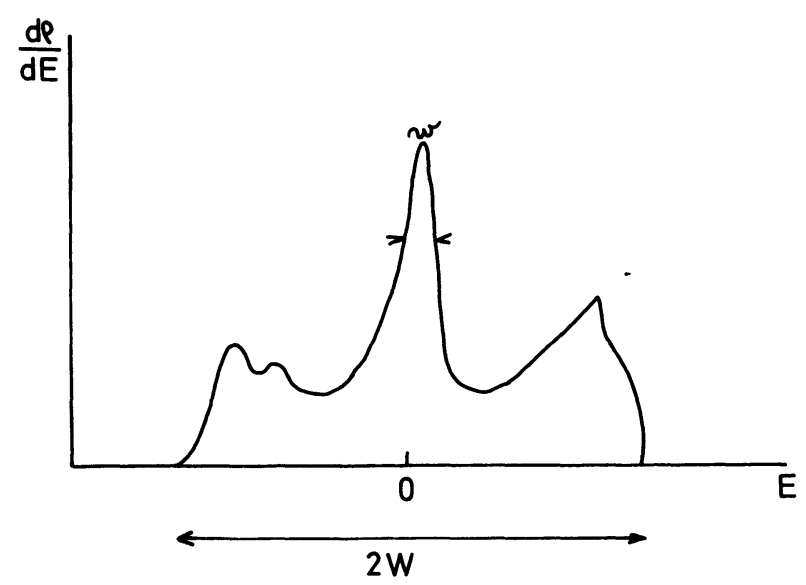

Fig. 11. - Local density of states on an adsorbed atom (schematic).

order of the sum of the transfer integrals $\sum_{i} \beta_{i}$ along the bonds of the adsorbed atom to the substrate. The corresponding life time $\tau_{\mathrm{v}}$ of a d electron on a volume atom is similarly such that

$$
W \simeq \hbar / \tau_{\mathrm{v}}
$$

is the sum of the transfer integrals $\beta_{i}$ along the bonds surrounding a volume atom. Thus $W$ is half the band width (Fig. 11).

Following this order of magnitude argument, one expects $w / W$ to be of the order of the ratio of the number of neighbours for an adsorbed atom and a volume atom. This should be of the order of $(5 \pm 1)^{-1}$ for all the surfaces considered if nearest neighbours alone are considered.

The resonant nature of the central peak is somewhat reinforced by the fact that the width of the virtual state is actually intermediary between $\sum_{i} \beta_{i}$ and $\left(\sum_{i} \beta_{i}\right)^{2} \frac{\mathrm{d} \rho}{\mathrm{d} E}$ : the density of surface states $\frac{\mathrm{d} \rho}{\mathrm{d} E}$ is especially low at the centre of the band for close packed surfaces of BCC and FCC structures; it is thus much less than $W^{-1}$.

There is some $a$ priori uncertainty in the use of these two types of formulae, which partly depends on the possible coherency of the effects of the various channels $\beta_{i}$ through which an electron on an adsorbed atom can jump back to the substrate. The directionality of the transfer integrals for the $d$ states and the contribution of second nearest neighbours in the BCC structure should alter but little these estimates.

The main results can be checked on the example of a simple s band and an adsorbed atom with one or two neighbours (Fig. 12a,b).

Let

$$
\mathscr{H}=\mathscr{H}_{0}+v
$$

be the hamiltonian of the problem, where

$$
\mathcal{H}_{0}=\beta \sum_{\substack{n \neq 0 \\ m \neq 0, \text { near to } n}}|n\rangle\left\langle m\left|+E_{0} \sum_{n}\right| n\right\rangle\langle n|
$$


and

$$
\begin{array}{r}
v=\beta(|0\rangle\langle 1|+| 1\rangle\langle 0|+| 0\rangle\langle 2|+ \\
+|2\rangle\langle 0|)
\end{array}
$$

$(|n\rangle$ is the sorbital on site $n)$.

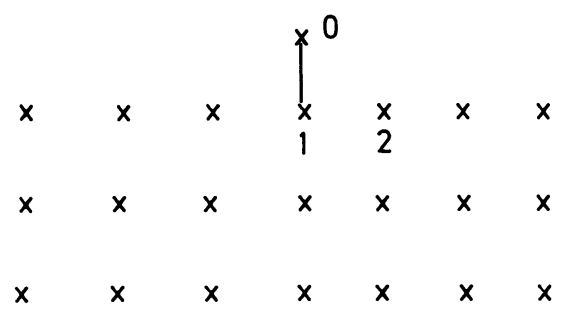

a)

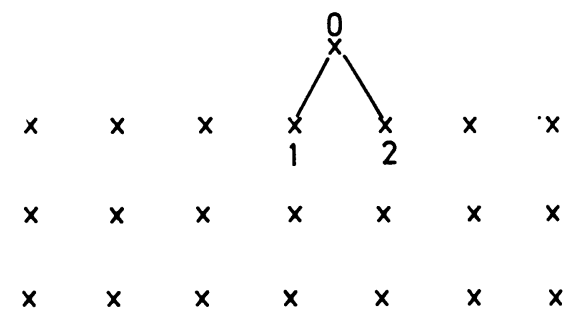

b)

FIG. 12. - Example of an adsorbed atom with one or two neighbours 1,2 (simple s band).

The site 0 is included in the second term of $\mathscr{H}_{0}$, and we shall take $E_{0}=0$ in the results, as before.

Introducing

$$
\begin{aligned}
G(z) & =\frac{1}{z-\mathscr{H}} \\
G_{0}(z) & =\frac{1}{z-\mathfrak{H}_{0}} \\
X(z) & =G(z) \frac{1}{G_{0}(z)},
\end{aligned}
$$

it is well known that the local density of states on the site 0 of the adsorbed atom is given by

$$
\frac{\mathrm{d} \rho_{0}}{\mathrm{~d} E}=\frac{1}{\pi} \operatorname{Im}\left\langle 0\left|G^{+}(E)\right| 0\right\rangle
$$

and the excess density of volume states introduced by the adsorbed atom is

$$
\delta n(E)=\frac{1}{\pi} \frac{\mathrm{d} \eta}{\mathrm{d} E}+\frac{\mathrm{d} \delta(E)}{\mathrm{d} E}=\frac{1}{\pi} \frac{\mathrm{d} \eta^{\prime}}{\mathrm{d} E}
$$

with

$$
\eta=\arg \ln \operatorname{det} X^{+}(E) .
$$

The notation

$$
0^{+}(E)=\lim _{\varepsilon \rightarrow+0} 0(E+i \varepsilon)
$$

is used.

Straightforward computations give

$$
\frac{\mathrm{d} \rho_{0}}{\mathrm{~d} E}=\frac{1}{\pi} \frac{\beta^{2} \operatorname{Im} \mathcal{G}}{\left(E-\beta^{2} \operatorname{Re} \mathcal{G}\right)^{2}+\beta^{4}(m \mathcal{G})^{2}}
$$

and

$$
\operatorname{tg} \eta^{\prime}=-\frac{\beta^{2} \operatorname{Im} \mathcal{G}}{E-\beta^{2} \operatorname{Re} \mathcal{S}}
$$

where for one neighbour (Fig. 12a)

$$
\mathcal{G}=\left\langle 1\left|G_{0}^{+}\right| 1\right\rangle
$$

and for $q$ neighbours (Fig. $12 b$ for $q=2$ )

$$
\mathcal{G}=\sum_{i, j=1 \text { to } q}\left\langle i\left|G_{0}^{+}\right| j\right\rangle .
$$

For substrates with alternate structures [19] (such as (100) or (110) surfaces of BCC structures, if nearest neighbours are concerned), the surface density of states $\operatorname{Im}\left\langle 1\left|G_{0}^{+}(E)\right| 1\right\rangle$ is even in energy, and its transform $\operatorname{Re}\left\langle 1\left|G_{0}^{+}(E)\right| 1\right\rangle$ is odd. A development for small energies $E$ of $(28,(29)$ gives, for one neighbour :

$$
\begin{aligned}
& \frac{1}{\pi} \frac{\mathrm{d} \eta^{\prime}}{\mathrm{d} E}=\frac{\mathrm{d} \rho_{0}}{\mathrm{~d} E} \simeq \frac{1}{\pi} \times \\
& \quad \times \frac{\beta^{2} \operatorname{Im} \mathcal{G}(0)}{\left[1-\beta^{2}\left(\frac{\mathrm{d} \operatorname{Re} \mathcal{G}}{\mathrm{d} E}\right)_{0}\right] E^{2}+\beta^{4}(\operatorname{Im} \mathcal{G}(0))^{2}} .
\end{aligned}
$$

The width of the lorentzian is

$$
w \simeq \beta^{2} \frac{\operatorname{Im} \mathcal{G}(0)}{1-\left(\frac{\mathrm{d} \operatorname{Re} \mathcal{G}}{\mathrm{d} E}\right)_{0}} .
$$

It is easy to check that

$$
\left(\frac{\mathrm{d} \operatorname{Re} \mathcal{G}}{\mathrm{d} E}\right)_{0} \simeq \frac{1}{W^{2}} \text { and } \operatorname{Im} \mathcal{G}(0) \simeq \frac{\pi}{W}
$$

Thus, for one neighbour

$$
w \simeq \pi \frac{\beta^{2}}{W} \ll W .
$$

The approximations leading from (28) to (32) are thus valid, and a resonant central peak exists. The egality of $\frac{1}{\pi} \frac{\mathrm{d} \eta^{\prime}}{\mathrm{d} E}$ and of $\frac{\mathrm{d} \rho_{0}}{\mathrm{~d} E}$ shows that the excess density of states produced by the surface is essentially localized on the adsorbed atom. This would not be the case if the adsorbed atom was of a chemical nature different from the substrate. 
This result is still approximately valid for non alternate structures such as the FCC or CPH. The resonant peak is somewhat shifted from the centre of the band, because $\operatorname{Im}\left\langle 1\left|G_{0}^{+}(E)\right| 1\right\rangle$ is no longer symmetrical, and thus $\operatorname{Re}\left\langle 1\left|G_{0}^{+}(E)\right| 1\right\rangle$ goes to zero at an energy different from zero. The equality of $\frac{1}{\pi} \frac{\mathrm{d} \eta^{\prime}}{\mathrm{d} E}$ and $\frac{\mathrm{d} \rho_{0}}{\mathrm{~d} E}$ is no longer exact. Eqs. (33) and (34) are then still approximately valid.

If there are $q$ neighbours, the same discussion applies where $\mathcal{G}$ is now given by (31). At the centre of the band $(E \simeq 0)$, the wave lengths of the electrons are of atomic dimensions. We expect therefore the contributions of the various terms $\left\langle i\left|G_{0}^{+}(E)\right| j\right\rangle$ to the imaginary (or real) part of $\mathcal{G}$ to be of similar amplitudes but possibly different signs. As a result,

$$
0<w<\frac{\pi(q \beta)^{2}}{W}
$$

where the lower limit would occur if the various channels were just in antiphase, and the upper one if they were in phase. In fact, neither of these extreme cases is realistic. A more reasonable estimate is obtained by assuming the $q$ channels as incoherent. $\mathcal{G}$ would just be multiplied by $q$, and

$$
w \simeq \frac{\pi q \beta^{2}}{W}
$$

As $W$ is not much larger than $\pi \beta$, this is not very different from the first estimate $w \simeq \sum_{i} \beta_{i}$.

3.2 INTERFERENCE EFFECTS BETWEEN VIRTUAL BOUND LEVELS. - 3.2.1 Following the idea of the previous paragraph, one way of describing a flat but not so close packed surface is as produced by a collection of adsorbed atoms, regularly spaced.

This seems particularly reasonable when the atoms on the surface plane have only second nearest neighbours in the surface plane, and all their first neighbours in the underlying substrate : (this is the case of the (100) surface of the BCC structure). This picture might also apply more generally when surface atoms have much fewer effective nearest neighbours than volume atoms, and especially few within the surface plane.

A simple count of nearest neighbours shows less difference between close-packed and not so close packed surfaces for the FCC structure than for the BCC one, as table III shows. But the difference between close packed and non close packed surfaces in the FCC structure is increased, at least for some d states, if their anisotropy is taken into account. Thus for the (100) surface of the FCC structure, table II shows that the $x_{1} x_{2}, x_{2} x_{3}, x_{3} x_{1}$, orbitals have small transfer integrals with their nearest neighbours in the surface plane. The same applies, to a lesser extent, to the $x_{33}$ orbitals, but least to the $x_{1}^{2}-x_{2}^{2}$ ones.

In conclusion, the flat but not so close packed surfaces of the BCC and FCC structures can be reasonably thought of as a collection of adsorbed atoms for all but the $x_{1}^{2}-x_{2}^{2}$ orbitals in the (100) surface of the FCC structure.

3.2.2 In these cases where the description in terms of adsorbed atoms might apply, a virtual bound $\mathrm{d}$ state effect, thus a narrow central peak of surface density of states, is expected only if two supplementary conditions are fulfilled :

1) Few or weak interactions with the substrate.

2) Weak coherence effects, i.e. weak indirect interactions between virtual $\mathrm{d}$ states [20].

1) The first condition has been stressed above for isolated virtual d states. It is not easy to gauge exactly in specific cases.

It is however clear from table I that, in the (100) surface of the BCC structure, interactions with atoms of plane 2 or 3 are large for none of the $d$ orbitals. They are especially weak for the $x_{1}^{2}-x_{2}^{2}$ orbitals. Interactions with atoms of plane 2 are also not very large for all $\mathrm{d}$ orbitals in the (100) surface of the FCC structure (table II). It can be expected that similar results apply to the (110) surface of the FCC structure.

2) The second condition is obviously related to the first one. Coherency effects between the $d$ states of the various atoms of the last plane will lead to a broadening of the central peak. These are small only if there is little or no direct bonding within the surface plane, but also if indirect interactions through bonding with the substrate are weak. The second condition does not therefore introduce any supplementary limitation of substance, and can be neglected.

This last result can be shown mathematically on a simple s band model. Consider a family of adsorbed atoms $i^{\prime}$, each interacting with only one atom $i$ of the

TABLE III

Number of first neighbours in cubic structures

Structure

Surface

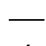

Number in surface plane

Total number for surface atom

Number for volume atom
4
$\mathrm{BCC}$

6

8

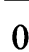

0

$(100)$
-
0
4
8

FCC

(100)

-
8
12

-
7
12


substrate (Fig. 13). These adsorbed atoms are assumed to have the same atomic energy as the substrate and to be regularly spaced, on a two-dimensional lattice which is a multiple of that of the substrate surface; the transfer integral $\beta$ along the $i i^{\prime}$ bonds is not necessarily

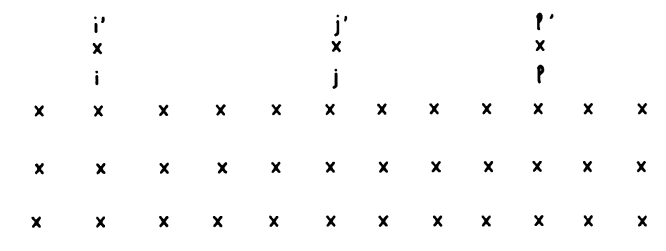

FIG. 13. - Coherency effects between adsorbed atoms.

the same as between the atoms of the substrate. We can introduce the Bloch functions

$$
\left|k_{P}\right\rangle=\frac{1}{\sqrt{N}} \sum_{i \in P} \exp i \mathbf{k} \mathbf{R}_{i}|i\rangle
$$

where the summation is on the $N$ sites $i$ of the $P$ th plane of the substrate, and $\mathbf{k}$ is a vector in the plane, taken in the first Brillouin of the substrate surface

$$
|L\rangle=\frac{1}{\sqrt{N^{\prime}}} \sum_{i^{\prime}} \exp i \mathbf{L} \mathbf{R}_{i}\left|i^{\prime}\right\rangle
$$

where summation is on the $N^{\prime}$ sites of adsorbed atoms $i^{\prime} . \mathbf{L}$ is a vector of the surface plane, defined within a reciprocal lattice vector $\mathbf{K}$ of the adsorbed lattice, in the reduced Brillouin zone scheme of the substrate surface : the number of such vectors $\mathbf{K}$ to take into account is $N / N^{\prime}$.

Taking

$$
\mathscr{H}=\mathscr{H}_{0}+v
$$

with

$$
v=\beta \sum_{i, i^{\prime}}\left(|i\rangle\left\langle i^{\prime}|+| i^{\prime}\right\rangle\langle i|\right)
$$

and $\mathscr{H}_{0}$ the hamiltonian of the substrate, the same definitions (22) to (27) for $\operatorname{tg} \eta$ and

$$
\frac{\mathrm{d} \rho_{i}}{\mathrm{~d} E}=\operatorname{Im}\left\langle i\left|G^{+}\right| i\right\rangle
$$

lead for $\beta$ small and to the same formulae (28), (29), with now

$\mathcal{G}=\frac{1}{N^{\prime}} \sum_{L, \boldsymbol{K}}\left\langle L\left|G_{0}^{+}(E)\right| L+K\right\rangle=\left\langle i\left|G_{0}^{+}(E)\right| i\right\rangle$.

In other words, to second order in $\beta / W$, where $W$ is the volume band width, the local density of states on the adsorbed atoms is the same as if they were isolated.

In conclusion, a central peak in the surface density of states can originate from practically non interacting virtual $\mathrm{d}$ levels of the surface atoms if two conditions are fulfilled together : weak intraplane and interplane interactions.

The condition of weak intraplane interactions is not fulfilled for the most closepacked surfaces considered : (111) in FCC, (110) in BCC; (0001) in HCP. And indeed no central peak has been computed or observed in such cases.
In other simple flat but less closepacked surfaces, the condition of weak intraplane interactions is fulfilled for most orbitals; the condition of weak interplane interactions seems to be also reasonably fulfilled, so that virtual bound levels leading to central peaks are expected, and indeed computed or observed. This applies to all $\mathrm{d}$ orbitals for the (100) surface of the BCC structure, and especially to the $x_{1}^{2}-x_{2}^{2}$ one, which has weak inter as well as intraplane interactions. It also applies to all but the $x_{1}^{2}-x_{2}^{2}$ orbitals for the (100) surface of the FCC structure (this last has too strong intraplane interactions). It probably applies also to the (110) surface of the FCC structure.

All these conclusions are in agreement with full LCAO computations and with photoemission experiments on Mo and W. There seems therefore to be a clearcut difference between surfaces : in the most closepacked ones, atoms mostly interact within the surface plane, and two-dimensional van Hove anomalies might have some meaning; but they are mixed with the peaks of the volume density of states; not so closepacked surfaces are best described as a collection of adsorbed atoms, with their resonant $d$ orbitals leading to nearly independent virtual bound states effects in the centre of the band.

This last case should obviously apply to atomically rough surfaces, as produced in some stages of condensation from vapour, or possibly at equilibrium near to the melting point.

Computations on steps of atomic height seem to show the same difference : non closepacked steps seem to present a central peak effect (eventually split by spin orbit); close packed steps present no central peak for the $\mathrm{d}$ orbital which can make a $\sigma$ band along the step [21].

The author acknowledges with thanks many stimulating discussions and corrections from A. Blandin, M. C. Desjonquères and L. Salem.

\section{APPENDIX A}

Mixed $x_{33}$ and $x_{1}^{2}-x_{2}^{2}$ subbands on the (100) surface plane of a cubic crystal. - We denote by $\left|i^{\prime}\right\rangle$ and $\left|i^{\prime \prime}\right\rangle$ respectively the (normalized) $x_{1}^{2}-x_{2}^{2}$ and $x_{33}$ orbitals on site $i$. The wave function of energy $E(\mathbf{k})$ can be written

$$
|\psi\rangle=\sum_{i} \mathrm{e}^{i \mathbf{k} \mathbf{R}_{i}}\left(A^{\prime}\left|i^{\prime}\right\rangle+A^{\prime \prime}\left|i^{\prime \prime}\right\rangle\right)
$$

The reduced LCAO hamiltonian for the same subbands can read

$$
\begin{aligned}
\mathbf{H}= & \sum_{\substack{i \\
j \text { near } i}}\left[\alpha^{\prime}\left|i^{\prime}\right\rangle\left\langle j^{\prime}\left|+\alpha^{\prime \prime}\right| i^{\prime \prime}\right\rangle\left\langle j^{\prime \prime}\right|\right]+ \\
& +\gamma \sum_{\substack{i \in n \\
j \in n \text { near } i}}\left(\left|i^{\prime}\right\rangle\left\langle j^{\prime \prime}|+| i^{\prime \prime}\right\rangle\left\langle j^{\prime}\right|\right) \\
& -\gamma \sum_{\substack{i \in n \\
j \in n \pm 1 \text { near } i}}\left(\left|i^{\prime}\right\rangle\left\langle j^{\prime \prime}|+| i^{\prime}\right\rangle\left\langle j^{\prime}\right|\right) .
\end{aligned}
$$

Number $n$ denotes the atomic row considered. 
The solution of $\mathbf{H}|\psi\rangle=E|\psi\rangle$ is given by

$$
\left|\begin{array}{ll}
2 \alpha^{\prime}\left(\cos k_{1} a+\cos k_{2} a\right)-E & 2 \gamma\left(\cos k_{1} a-\cos k_{2} a\right) \\
2 \gamma\left(\cos k_{1} a-\cos k_{2} a\right) & 2 \alpha^{\prime \prime}\left(\cos k_{1} a+\cos k_{2} a\right)-E
\end{array}\right|=0
$$

hence

$$
E=\left(\alpha^{\prime}+\alpha^{\prime \prime}\right)\left(\cos k_{1} a+\cos k_{2} a\right) \pm\left[\left(\alpha^{\prime}-\alpha^{\prime \prime}\right)^{2}\left(\cos k_{1} a+\cos k_{2} a\right)^{2}+4 \gamma^{2}\left(\cos k_{1} a-\cos k_{2} a\right)^{2}\right]^{1 / 2} .
$$

Here

$$
\begin{aligned}
\alpha^{\prime}+\alpha^{\prime \prime} & =\sigma+\delta \\
\alpha^{\prime}-\alpha^{\prime \prime} & =\frac{\sigma-\delta}{2} \text { hence }\left(\alpha^{\prime}-\alpha^{\prime \prime}\right)^{2}=\left(\frac{\sigma-\delta}{4}\right)^{2} \\
4 \gamma^{2} & =\frac{3(\sigma-\delta)^{2}}{4}
\end{aligned}
$$

$k_{1}$ and $k_{2}$ are the components of $\mathbf{k}$, taken between $\pm \pi / a$.

Van Hove anomalies are obtained by

$$
0=\frac{\partial E}{a \partial k_{1}}=\left[-\left(\alpha^{\prime}+\alpha^{\prime \prime}\right) \mp \frac{\left(\alpha^{\prime}-\alpha^{\prime \prime}\right)^{2} u+4 \gamma^{2} v}{D}\right] \times
$$

$0=\frac{\partial E}{a \partial k_{2}}=\left[-\left(\alpha^{\prime}+\alpha^{\prime \prime}\right) \mp \frac{\left(\alpha^{\prime}-\alpha^{\prime \prime}\right)^{2} u-4 \gamma^{2} v}{D}\right] \times$ $\times \sin k_{2} a$

with

$$
\begin{aligned}
u & =\cos k_{1} a+\cos k_{2} a \\
v & =\cos k_{1} a-\cos k_{2} a \\
D^{2} & =\left(\alpha^{\prime}-\alpha^{\prime \prime}\right)^{2} u^{2}+4 \gamma^{2} v^{2} .
\end{aligned}
$$

Such a van Hove anomaly is at the centre of the band if furthermore

$$
E=0 .
$$

The system (A.1) has four types of solutions :

1) $k_{1}=k_{2}=0$.

2) $k_{2}=0$

$\left.-\left(\alpha^{\prime}+\alpha^{\prime \prime}\right) \mp \frac{\left(\alpha^{\prime}-\alpha^{\prime \prime}\right)^{2} 4+4 \gamma^{2} v}{D}=0\right\}$ or vice versa

3)

$$
\begin{aligned}
-\alpha^{\prime}+\alpha^{\prime \prime} \mp \frac{\left(\alpha^{\prime}-\alpha^{\prime \prime}\right)^{2} u+4 \gamma^{2} v}{D}= \\
\quad=-\left(\alpha^{\prime}+\alpha^{\prime \prime}\right) \mp \frac{\left(\alpha^{\prime}-\alpha^{\prime \prime}\right)^{2} u-4 \gamma^{2} v}{D}=0 .
\end{aligned}
$$

It is easy to check that solutions 1) and 2) are not compatible with (A.2).
Solution (3) leads to

$$
\begin{aligned}
& \frac{u}{D}=\mp \frac{1}{\alpha^{\prime}-\alpha^{\prime \prime}} \\
& \frac{v}{D}=0 .
\end{aligned}
$$

The second equation leads to $v=0$, thus

$$
k_{1}=k_{2} \text {. }
$$

The first equation is then equivalent to the definition of $D$. The condition $E=0$ leads then to $\cos k_{1} a=0$, or

$$
k_{1} a= \pm \frac{\pi}{2}
$$

In the vicinity of $k_{1} a=k_{2} a=\frac{\pi}{2}$, one can develop $E(\mathbf{k})$ in function of $\varepsilon_{i}$ where :

$$
k_{i} a= \pm \frac{\pi}{2}+\varepsilon_{i}
$$

One finds :

$$
E=\mp\left(\frac{\sigma+\delta}{\sqrt{2}}\right) \eta_{1} \pm \frac{\sigma-\delta}{\sqrt{2}} \sqrt{\eta_{1}^{2}+3 \eta_{2}}
$$

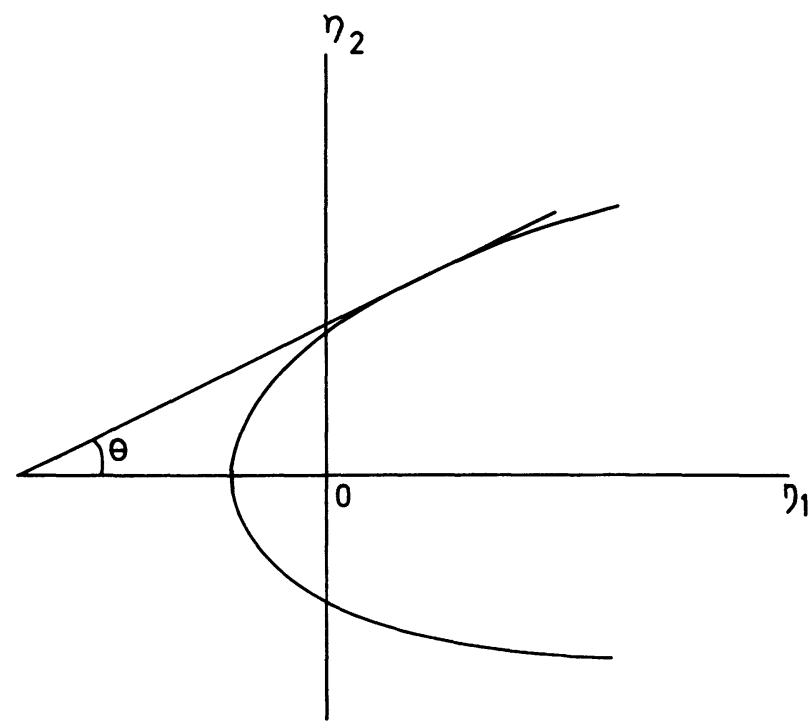

Fig. A.1. - Constant energy curves near a singular point. 
with

$$
\begin{aligned}
& \eta_{1}=\frac{\varepsilon_{1}+\varepsilon_{2}}{\sqrt{2}} \\
& \eta_{2}=\frac{\varepsilon_{2}-\varepsilon_{1}}{\sqrt{2}} .
\end{aligned}
$$

Neglecting $\delta$, eq. (A.3) becomes the equation of a parabola (Fig. A.1) :

$$
\eta_{1}=\mp \frac{E}{\sqrt{2} \sigma} \pm \frac{3}{2 \sqrt{2}} \sigma \eta_{2}^{2} .
$$

The density of states becomes :

$$
n(E)=\int \frac{\mathrm{d} \eta_{1}}{\cos \theta\left|\nabla_{k} E\right|}
$$

where

$$
\operatorname{tg} \theta=\frac{\mathrm{d} \eta_{2}}{\mathrm{~d} \eta_{1}} \simeq \pm \frac{\sqrt{2} E}{3 \eta_{2}}
$$

Expliciting $\nabla_{k} E$, one gets in the vicinity of the critical point :

$$
n(E)=\frac{E^{2}}{9 \sigma}\left(\frac{\operatorname{sh} 2 u}{2}+u\right)
$$

where

$$
\operatorname{sh} u=\frac{3 \eta_{2}}{\sqrt{2} E}
$$

This density remains finite when $E \rightarrow 0$.

\section{References}

[1] Haydock, R. and Kelly, M. J., Surf. Sci. 38 (1973) 139.

[2] Penn, D. R., Surf. Sci. 38 (1973) 333.

[3] Desjonquères, M. C. and Cyrot-Lackmann, F., J. Physique Lett. 36 (1975) L-45.

[4] Desjonquères, M. C. and Cyrot-Lackmann, F., J. Phys. F. Metal Physics 5(1975) 1368.

[5] Desjonquères, M. C. and Cyrot-Lackmann, F., Phys. Rev. submitted (1975)

[6] Schrieffer, J. R. and Soven, P., Phys. Today (1975) 24.

[7] Sturm, K. and Feder, R., Solid State Commun. 14 (1974) 1317.

[8] Kasowski, R. V., Solid State Commun. 17 (1975) 179.

[9] Plummer, E. W. and Gadzuk, J. W., Phys. Rev. Lett. 25 (1970) 1493.

[10] Feuerbacher, R. B. and Fitton, B., Phys. Rev. Lett. 29 (1972) $786 ; 30$ (1973) 923.

[11] Waclawski, B. J. and Plummer, E. W., Phys. Rev. Lett. 29 (1972) 783.
[12] Al Khoury Nemeh, E., Cinti, R. C. and Hudson, J. B., J. Physique Lett. 35 (1974) L-179.

[13] Al Khoury Nemeh, E., Thèse Grenoble (1975).

[14] Kalkstein, D. and Soven, P., Surf. Sci. 38 (1973) 139.

[15] Ducastelle, F., Thèse Orsay (1972).

[16] Grimley, T. B., Proc. Phys. Soc. 90 (1971) 751.

[17] Newns, D. M., Phys. Rev. B 1 (1970) 3304.

[18] Lyo, S. K. and GoMER, R., Theory of chemisorption in Topics in Applied Physics 4 Interactions on Metal Surfaces (Ed. R. Gomer Springer Berlin) 1975, p. 41.

[19] Friedel, J. and Lannoo, M., J. Physique 34 (1973) 483.

[20] Einstein, T. L. and SChriefFer, J. R., Phys. Rev. B 7 (1973) 3629.

[21] Desjonquères, M. C. and Cyrot-LackmanN, M. C. (1975) to be submitted. 